\title{
Direction Selective Neurons Responsive to Horizontal Motion in a Crab Reflect an Adaptation to Prevailing Movements in Flat Environments
}

\author{
${ }^{\circledR}$ Florencia Scarano, ${ }^{1}{ }^{\circledR}$ Daniel Tomsic, ${ }^{1,2}$ and ${ }^{\circledR}$ Julieta Sztarker ${ }^{1,2}$ \\ ${ }^{1}$ Instituto de Fisiología, Biología Molecular y Neurociencias (IFIBYNE) CONICET, Universidad de Buenos Aires, Buenos Aires 1428, Argentina, and \\ ${ }^{2}$ Departamento de Fisiología, Biología Molecular y Celular Dr Héctor Maldonado, Facultad de Ciencias Exactas y Naturales, Universidad de Buenos \\ Aires, Buenos Aires 1428, Argentina
}

\begin{abstract}
All animals need information about the direction of motion to be able to track the trajectory of a target (prey, predator, cospecific) or to control the course of navigation. This information is provided by direction selective (DS) neurons, which respond to images moving in a unique direction. DS neurons have been described in numerous species including many arthropods. In these animals, the majority of the studies have focused on DS neurons dedicated to processing the optic flow generated during navigation. In contrast, only a few studies were performed on DS neurons related to object motion processing. The crab Neohelice is an established experimental model for the study of neurons involved in visually-guided behaviors. Here, we describe in male crabs of this species a new group of DS neurons that are highly directionally selective to moving objects. The neurons were physiologically and morphologically characterized by intracellular recording and staining in the optic lobe of intact animals. Because of their arborization in the lobula complex, we called these cells lobula complex directional cells (LCDCs). LCDCs also arborize in a previously undescribed small neuropil of the lateral protocerebrum. LCDCs are responsive only to horizontal motion. This nicely fits in the behavioral adaptations of a crab inhabiting a flat, densely crowded environment, where most object motions are generated by neighboring crabs moving along the horizontal plane.
\end{abstract}

Key words: crabs; direction selectivity; environmental adaptations; intracellular recordings; lobula neurons; optic flow

Significance Statement

Direction selective (DS) neurons are key to a variety of visual behaviors including, target tracking (preys, predators, cospecifics) and course control. Here, we describe the physiology and morphology of a new group of remarkably directional neurons exclusively responsive to horizontal motion in crabs. These neurons arborize in the lobula complex and in a previously undescribed small neuropil of the lateral protocerebrum. The strong sensitivity of these cells for horizontal motion represents a clear example of functional neuronal adaptation to the lifestyle of an animal inhabiting a flat environment.

\section{Introduction}

Animals need to detect motion but also must be highly sensitive to its direction. The ability to perceive motion direction entails the presence of neurons responding differently to visual stimuli

Received Feb. 17, 2020; revised May 14, 2020; accepted May 16, 2020.

Author contributions: F.S., D.T., and J.S. designed research; F.S. performed research; F.S., D.T., and J.S. analyzed data; F.S., D.T., and J.S. wrote the paper.

This work was supported by the Agencia Nacional de Promoción Científica y Tecnológica Grants PICT 20160196 (to J.S.) and PICT 2016-1946 (to D.T.) and by the Universidad de Buenos Aires Ciencia y Tecnología Grant 20020170100254BA (to D.T.). We thank Dr. Martin Berón de Astrada for his valuable help with the intracellular staining protocol.

The authors declare no competing financial interests.

Correspondence should be addressed to Julieta Sztarker at sztarker@fbmc.fcen.uba.ar.

https://doi.org/10.1523/JNEUROSCI.0372-20.2020

Copyright $\odot 2020$ the authors moving in opposite directions. Such elements are generically called direction selective (DS) neurons.

DS neurons associated with optic flow analysis have been described in diverse arthropods (e.g., flies: Hausen, 1982a,b; Hausen and Egelhaaf, 1989; honeybees: DeVoe et al., 1982; Ibbotson, 1991; moths: Rind, 1983; locusts: Rind, 1990; hawkmoths: Wicklein and Varjú, 1999; praying mantis: Yamawaki, 2019; crabs: Sandeman et al., 1975). They usually have a wide receptive field, are very reactive to gratings in a particular axis (horizontal or vertical), respond with excitation to one motion direction (preferred direction) and with strong inhibition to the opposite one (null direction). They also show little adaption to stimulus repetition and convey information (directly or indirectly) to descending pathways commanding motor outputs (wing, neck, legs). 
On the other hand, less information is available from DS neurons involved in object detection or figure-ground discrimination. Neurons falling into this category appear to be more heterogeneous in their properties. They usually display a relatively small and ipsilateral receptive field, prefer small objects, and display a rapid adaptation on stimulus repetition. Among them are FD cells of blowflies (Egelhaaf, 1985; Warzecha et al., 1993), movement-sensitive neurons of butterflies (Swihart, 1968), DS neurons from hawk-moths (Collett, 1971, 1972), DS neurons from crayfish (Glantz, 2008), and MLG1 of crabs (Medan et al., 2007). Other examples are found within small-target-motion detectors (STMD), some of which present direction preference (Gilbert and Strausfeld, 1991; O'Carroll, 1993; Nordström et al., 2006; Barnett et al., 2007; Trischler et al., 2007). Most of this type of cells are described as DS neurons because they display a stronger response in one direction compared with the other, but they lack a proper inhibitory response in the null direction (Wiersma and Yanagisawa, 1971; O'Carroll, 1993; Nordström et al., 2006; Barnett et al., 2007; Medan et al., 2007; Glantz, 2008).

All animals have characteristics that evolved as adaptations to the specific environment that they inhabit. In particular, flat world arthropod species (e.g., crabs living in sandy beaches and mudflats or insects that live immediately above or below the water surface) show visual adaptations involving, among others, a particular disposition of acute zones in the eyes and specialized optokinetic sensitivity (for review, see Zeil et al., 1989). The DS neurons so far described in arthropods have been associated to specific behaviors (optokinetic responses, course control, tracking behaviors, detecting small objects). However, whether DS neurons can be shaped by the geometrical structure of the environment has never been investigated.

The crab Neohelice granulata is a renown model species in the field of neuroethology (Romano et al., 2006; Tomsic et al., 2009; Tomsic and Sztarker, 2019). Using in vivo intracellular recordings, we have previously characterized diverse neurons of this crab involved in visual processing (Berón de Astrada et al., 2001, 2009; Berón de Astrada and Tomsic, 2002), including high-integration level neurons of the lobula (the third optic neuropil), generically known as lobula giant (LG) neurons, which reflect many properties of the crab's escape behavior including the intensity and temporal dynamics of response to visual danger stimuli (black bars moving overhead and looming stimuli that represent potential predators attacks), seasonal response changes, learning-induced changes, etc. (Tomsic et al., 2003; Sztarker and Tomsic, 2008, 2011; Medan et al., 2015). Here, we describe in this crab a new type of neurons with remarkable directional preference that includes strong inhibition in the null direction. These are large tangential cells with broad arborizations in the lobula complex, for which we called them lobula complex directional cells (LCDCs). We discuss their possible role in object detection and optic flow analysis and their selectivity for horizontal motion in relation to the lifestyle of crabs inhabiting a flat environment.

\section{Materials and Methods}

\section{Animals}

Animals were adult male $N$. granulata crabs measuring $2.7-3.0 \mathrm{~cm}$ across the carapace, weighing $\sim 17 \mathrm{~g}$, collected in the rias (narrow coastal inlets) of San Clemente del Tuyú, Argentina. The crabs were transported to the laboratory, where they were maintained in plastic tanks $(35 \times 48$ $\times 27 \mathrm{~cm}$ ) filled to $2-\mathrm{cm}$ depth with artificial seawater to a density of 20 crabs per tank. Water used in tanks and other containers during the experiments was prepared using Coral Pro Salt for Marine Aquarium (manufacturer Red Sea) to reach a salinity of $10-14 \%$, pH 7.4-7.6. The holding room had transparent polycarbonate walls and roof, so the animals were exposed to natural light, and the temperature was maintained between $22^{\circ} \mathrm{C}$ and $24^{\circ} \mathrm{C}$. The experiments were run between 9 A.M. and 8 P.M.

\section{Electrophysiology}

Intracellular recordings from neurons in the optic lobes were performed in the intact living animal according to methods previously described (Berón de Astrada and Tomsic, 2002). Briefly, the crab was firmly held in an adjustable clamp. The eyes were cemented to the carapace at an angle of $\sim 50^{\circ}$ from the horizontal plane, which correspond to their typical seeing position. A tangential cut performed with a sharp scalpel was made to remove a small section of cuticle $(\sim 500 \mu \mathrm{m}$ in diameter) from the tip of the eyestalk without causing damage to the ommatidial area. A glass microelectrode was then positioned and advanced through the opening in the cuticle. A ground electrode consisting of a silver wire was inserted through a small hole in the dorsal carapace. The clamped crab was positioned in the center of the monitor configuration and held in position using a magnetic holding device inside the Faraday cage.

Microelectrodes (borosilicate glass; $1.2 \mathrm{~mm}$ outer diameter, $0.68 \mathrm{~mm}$ inner diameter) were pulled on a Brown-Flaming micropipette puller (P-97; Sutter Instrument) yielding tip resistances of 40-80 $\mathrm{M} \Omega$ when filled with $3 \mathrm{M} \mathrm{KCl}$. A bridge balance amplifier was used for intracellular recordings (AxoClamp 2B; Molecular Devices). The output of the amplifier was monitored on an analog oscilloscope, digitized at $10 \mathrm{kHz}$ (Digidata 1320, Molecular Devices), and recorded with Clampex (from pClamp9 suite, Molecular Devices) for subsequent off-line analysis using Clampfit (from pClamp 9, Molecular Devices).

The impalements were performed blindly. The procedure implies lowering the electrode and evaluating the responses of impaled cells to visual stimulation. Based on previous characterizations of different neuronal types of the optic neuropils, cell responses to a flash of light and to a moving stimulus allow to rapidly recognize the neuropil from which they are being recorded (Tomsic and Sztarker, 2019). In the crab visual system, highly motion sensitive neurons arise in the third optic neuropil (Berón de Astrada and Tomsic, 2002; Medan et al., 2007). Thus, to search for motion directional cells we rapidly descended the microelectrode into this region. Once in there, we tested every cell that we impaled with the bar moving in both directions. The vast majority of cells ( $\sim 90 \%)$ did not show directional preference and were immediately abandoned to continue searching until a DS neuron was impaled. If a DS neuron could not be found in the term of $1 \mathrm{~h}$, we discarded the animal and started with a new one.

\section{Cell morphology}

Electrode tips were backfilled with 5\% Neurobiotin, 50 mм Tris buffer in $500 \mathrm{~mm} \mathrm{KCl}$ solution, backed up with $3 \mathrm{~m} \mathrm{KCl}$. Following characterization of their response to the visual stimuli, the cells were iontophoretically filled for $15-90 \mathrm{~min}$ using +1 to $+5 \mathrm{nA}$ current. During the injections, the neuron was periodically monitored, and its responsiveness and membrane potential were evaluated. Only one neuron per animal was injected. After iontophoresis, the tracer was allowed to diffuse for 2-4 $\mathrm{h}$ in the living animal. Then, the crab was anesthetized on icy water, and the optic lobe was dissected and immediately immersed in $4 \%$ paraformaldehyde in phosphate buffer ( $\mathrm{pH} 7.2$ ) to be fixed overnight. After five 20 min washes with PTA (PBS $0.1 \mathrm{~m}$, Triton X-100 2\% $\mathrm{v} / \mathrm{v}$, and sodium azide $0.1 \% \mathrm{wt} / \mathrm{vol}$; $\mathrm{pH} 7.4$ ), ganglia were incubated overnight with Streptavidin-Alexa Fluor 647 or Streptavidin-DyLight 594 $\left(1: 200 \mathrm{v} / \mathrm{v}\right.$ in buffer PTA) at $4^{\circ} \mathrm{C}$ with constant shaking, after which they were again washed five times with PTA. The ganglia were then dehydrated in ethanol series and cleared in methyl salicylate.

Cleared ganglia were imaged as whole mounts and scanned at 1.5- to $5-\mu \mathrm{m}$ intervals with a confocal microscope equipped with a helium/neon laser (Olympus Fluoview 1000). Images, saved as stacks, were adjusted for brightness and contrast, and three-dimensional reconstructions were obtained using Neurolucida software. 
Visual stimuli and experimental protocol

Computer-generated visual stimuli were projected on two computer screens (Samsung S20C300L) placed next to each other, forming an angle of $99^{\circ}$. The crab was located equidistant to the screens, at a distance of $27.4 \mathrm{~cm}$. During the first experiments, the crab was placed facing the pair of angled screens in such a way that it perceived visual stimulations on both of its fronto-lateral sides. Since all LCDCs recorded in this configuration responded only to the ipsilateral field, additional experiments were made with the crab rotated $90^{\circ}$, i.e., with the animal looking at the angle formed by the screens with the lateral pole of the recorded eyestalk. This allowed to present the stimuli covering the antero-lateral and postero-lateral fields of view of the recording side (ipsilateral side). The screens and the crab were housed inside a Faraday cage completely covered with an opaque felt to prevent outside visual stimuli from reaching the animal. After a neuron has been impaled, a black curtain was lowered in the front part of the cage. Visual stimuli were generated with a single PC, using commercial software (Presentation 5.3, Neurobehavioral Systems). The signal generated by the PC was first split and then sent to a switcher for selecting between the two screens. From each selector the video signal could be rapidly turned on and off. When the selector was in the off position, a second computer was used to feed a uniform white background that remained on when no stimulus was presented on the monitor. The selector and other control systems used during the experiments were located outside the Faraday cage. In this way, the experimenter could choose which screen/s showed the stimulus or just the background at any time without distressing the animal.

Visual stimuli consisted of a black bar $(4 \times 21 \mathrm{~cm}$; retinal subtended angle at the center of the screen $\left.8.6^{\circ} \times 43.3^{\circ}\right)$ moving over a white background. The stimulus was presented separately on each screen, moving rightward, leftward, downward, or upward, always perpendicular to its major axis, at a speed of $11 \mathrm{~cm} / \mathrm{s}$. The distances covered in horizontal and vertical translations were 37 and $17 \mathrm{~cm}$ (spanning $\operatorname{arcs}$ of $69.8^{\circ}$ and $35.6^{\circ}$ ), respectively. The size and speed were chosen based on previous studies showing that these stimuli effectively evoked behavioral responses (Scarano and Tomsic, 2014). After presenting the four cardinal directions to 15 cells, it was evident that the cells only responded to horizontal motion, therefore for the rest of the recordings (51 cells recorded in total), only rightward and leftward stimuli were used. To avoid the effect of appearing and disappearing from behind the borders of the screen, the stimulus started and ended its trajectory from positions separated $1 \mathrm{~cm}$ from the screen borders. Stimuli were stationary for $30 \mathrm{~s}$ before movement onset. The order of stimulation (screen and movement direction) was randomized, and the intertrial interval was 1 min to curtail habituation. We also used a panoramic grating of equally separated vertical black and white bars of the same size $(4 \times 21 \mathrm{~cm})$ and speed $(11 \mathrm{~cm} / \mathrm{s})$ as the single bar stimulus (BS), moved horizontally to the right or to the left during $15 \mathrm{~s}$. The pattern was presented simultaneously and coherently on the two monitors. The grating was incorporated late in our experiments, therefore, the responses to this stimulus were tested only in $50 \%$ of the LCDCs that we recorded.

The complete stimulation series in a single experiment took at least $15 \mathrm{~min}$ to complete. During this time, the animal occasionally moved its legs, which sometimes caused the loss of the cell impalement before the end of the experiment. Data from incomplete series, however, provided valuable information and were included in some analyses.

\section{Data analysis}

The motion sensitivity of the LCDCs to the four cardinal directions was assessed by measuring the neuronal input and output signals recorded during stimuli presentations. For the input, we considered the EPSP and the IPSP, whereas for the output signals we measured the number of elicited action potentials (APs). In order to measure the EPSP component, APs were digitally removed from the records by using a Gaussian lowpass filter implemented in Clampfit. The intensity of the EPSP and IPSP response components was estimated by the maximum membrane change (positive or negative, respectively) reached during the stimulus presentation with respect to the previous 2 -s average membrane potential. The output signal was considered as the number of APs elicited by the stimulus divided by the duration of the trial (horizontal direction:
$3.36 \mathrm{~s}$; vertical direction: $1.7 \mathrm{~s}$ ). Given the difference in spontaneous activity among cells, the spontaneous firing rate was subtracted. The spontaneous firing rate was estimated for each trial from the 2-s period preceding the stimulus onset.

To quantify the response preference of the neurons to the motion of a single moving bar (BS) versus to the motion of a panoramic grating stimulus (GS), we calculated an object preference index (OPI). The OPI was calculated using the APs elicited during the second of maximal response to the bar (APBS) and the number of APs triggered during the first second of motion of the grating (which always held the strongest response; $A P G S$ ). The rationale for not using the first second of response to the bar, and instead choosing the time window of maximal response in each cell, is because the bar entered into the receptive field of different cells at different times:

$$
\mathrm{OPI}=\frac{\mathrm{APBS}-\mathrm{APGS}}{\mathrm{APBS}+\mathrm{APGS}}
$$

The index varies from 1 to -1 corresponding to exclusive bar or grating preference, respectively. Values around 0 represent no preference, i.e., a similar response triggered by the bar or the grating.

To quantify the change in response during the sustained motion of a GS, we calculated an index of response persistence to the grating (IRPG), where $R_{i}$ was the initial response measured as the number of APs triggered in the first $2 \mathrm{~s}$ of response, and $\mathrm{R}_{\mathrm{f}}$ was the final response measured as the number of APs triggered in the last $2 \mathrm{~s}$ :

$$
\mathrm{IRPG}=\frac{1-\left(\mathrm{R}_{\mathrm{i}}-\mathrm{R}_{\mathrm{f}}\right)}{\mathrm{R}_{\mathrm{i}}} .
$$

The index value 0 stands for responses that completely vanished after $15 \mathrm{~s}$ of continuous stimulation, 1 for responses maintained unaltered for the whole period, and above 1 for responses that incremented along the trial.

Only neurons that responded with a minimum of five spikes (over spontaneous activity) to the preferred direction were included for analyses. One-way repeated-measures ANOVAs with direction as factor of analysis were performed to establish direction preference of LCDCs. Tukey's multiple comparisons post hoc tests were used afterward. All statistical analyses were performed with GraphPad Prism (GraphPad Software). All data are presented as the mean \pm SEM. All experimental protocols were performed in accordance with relevant guidelines and regulations of the Universidad de Buenos Aires.

\section{Results}

\section{Differential response to the direction of a moving object}

We recorded intracellularly 51 neurons from 46 crabs with a marked selectivity for the direction of a moving object. The cells had different levels of spontaneous activity ranging from 0 to 19.5 Hz. They usually presented EPSPs and/or IPSPs both during spontaneous activity and during visual stimulation. Some neurons fired single spikes while others fired in bursts. The mean rest membrane potential was $-49.15 \pm 1.42 \mathrm{mV}(n=51)$. These cells had a relatively narrow receptive field $\left(\sim 50^{\circ}\right)$ always located in the ipsilateral field of vision. Unlike the majority of LG tangential cells previously characterized in crabs (Berón de Astrada and Tomsic, 2002; Medan et al., 2007), DS neurons presented no obvious response to a pulse of light nor to mechanosensory stimulation (tested by touching the legs with a paintbrush). All these cells responded with compound EPSPs and high-frequency discharges of APs to the bar moving in one direction (the preferred direction) and with sustained hyperpolarization in the opposite direction (null direction; Fig. 1). Because they were recorded in the lobula and also arborized in the lobula plate (later described in detail), we termed them LCDCs. The LCDCs showed a 

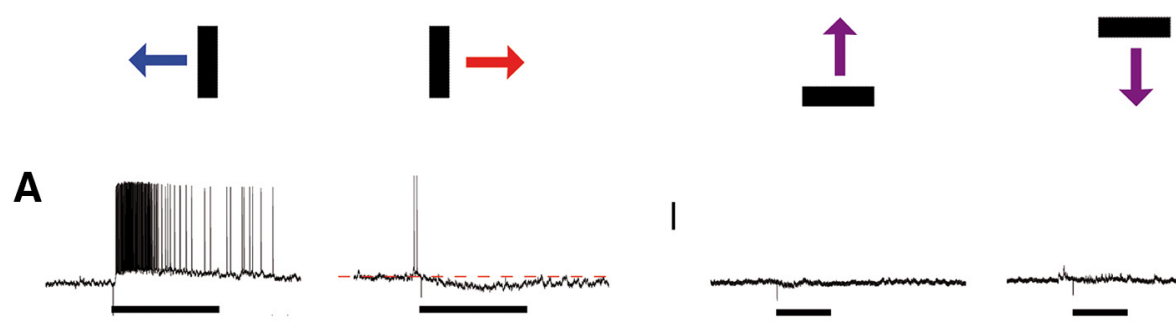

I

B
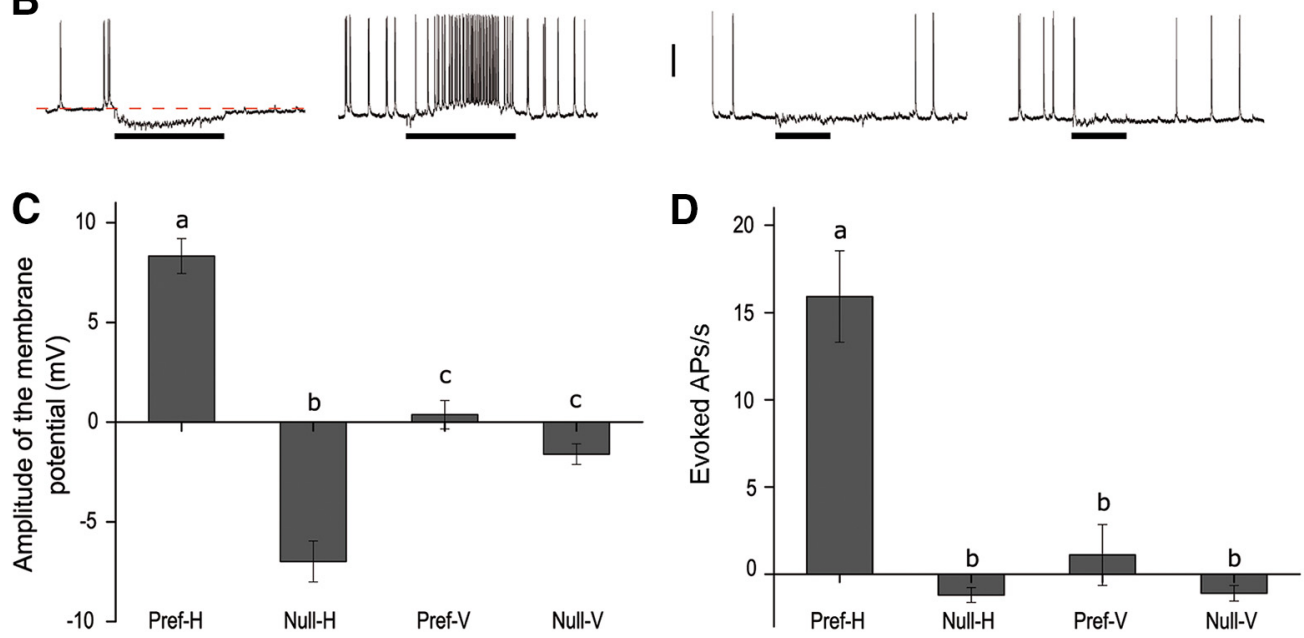

Figure 1. Response characteristics of $L C D C S . A, B$, Representative examples of the responses of two different cells. Each row $(\boldsymbol{A}, \boldsymbol{B})$ includes responses of the same neuron to the four cardinal directions of movement. The bars drawn above represent object motion in each column: back to front horizontal motion (blue), front to back horizontal motion (red), upwards and downwards, respectively. Both neurons respond to horizontal motion but show opposite preferred directions. Vertical scale bar: $15 \mathrm{mV}(\boldsymbol{A}), 20 \mathrm{mV}(\boldsymbol{B})$. C, D, Mean response of 15 neurons $(\boldsymbol{C}$, amplitude of the compound synaptic response; $\boldsymbol{D}$, evoked APs) triggered in the four cardinal directions ( $\mathrm{H}$ : horizontal motion; V: vertical motion). $\boldsymbol{C}$, When measuring the extent of the membrane potential responses, both the Pref-H and Null-H are significantly different $\left(F_{(3,39)}=63.56, p<0.0001\right.$; Tukey's multiple comparisons test: a vs b: $p<0.0001$; a vs c: $p<0.0001 ; \mathrm{b}$ vs $\left.\mathrm{c}: p<0.0001\right)$. $D$, When measuring the number of APs triggered per second (spontaneous activity was subtracted, see Materials and Methods), only one direction (Pref-H) evokes a significantly larger response $\left(F_{(3,42)}=28.22, p<0.0001\right.$; Tukey's multiple comparisons test a vs $\left.\mathrm{b}: p<0.0001\right)$. Black rectangles beneath the recordings indicate the period of motion of the bar in the direction indicated in the scheme above. Horizontal direction: 3.36 s; vertical direction: 1.7 s. Different letters denote significant differences. Error bars indicate SEM.

remarkable preference for horizontal motion. All cells tested with stimuli moving in the four cardinal directions responded to the displacement of the vertical bar moving horizontally but not to the horizontal bar moving vertically (Fig. 1). Recordings in Figure $1 A, B$ show two neurons with opposite preferred direction in the horizontal plane and no response to vertical motion in any direction. Figure $1 C, D$ shows averaged responses of 15 neurons that were stimulated in the four cardinal directions of motion. Figure $1 C$ shows the maximal magnitude of EPSP or IPSP elicited responses, which represent the excitatory or inhibitory inputs received by the cells during motion stimulation. Figure $1 D$ shows the number of elicited spikes per second, which represents the output signal conveyed by the neurons. For horizontally moving stimuli, depolarizing and hyperpolarizing responses were grouped as preferred (Pref-H) and null direction (Null-H), respectively. For vertically moving stimuli, responses were negligible in the two directions. However, to include these data into the analysis, in each neuron, we assigned as the preferred direction (Pref-V) that in which some depolarization could be observed and as Null-V the opposite direction. All LCDCs responded to the horizontal displacement of the stimulus in both directions. As showed in Figure $1 C$, both the preferred and null directions showed a significantly different effect $\left(F_{(3,39)}=63.56\right.$, $p<0.0001$; Tukey's multiple comparisons test: Pref-H vs NullH: $p<0.0001$; Pref-H vs Pref-V: $p<0.0001$; Null-H vs Null-V: $p<0.0001$; Pref-V vs Null-V: $p=0.3106$ ). Considering their output signal (Fig. 1D) LCDCs exhibit a remarkable degree of directional preference $\left(F_{(3,42)}=28.22, p<0.0001\right.$; Tukey's multiple comparisons test: Pref-H vs Null-H: $p<0.0001$; Pref-H vs Pref-V: $p<0.0001)$. Within the 15 LCDCs included in the analysis of Figure 1, nine preferred front to back motion and six back to front motion.

\section{Response preference}

DS cells of arthropods have been implicated in coding for optic flow field motion as well as in object motion. We wondered in which of these processes the LCDCs of crabs might be involved in. For this purpose, we compared the responses of LCDCs to the motion of a single bar and the motion of a panoramic grating that included that bar (Fig. 2A,B). All tested cells were excited with both stimuli when moved in one direction and inhibited in the opposite direction. No difference was found between the mean responses elicited by the two stimuli moved in the preferred direction during the 1-s time window of highest response (two-tailed paired $t$ test: $t_{(24)}=0.2617, p=0.7958$; Fig. 2C). Such result could be explained because all neurons responded similarly to both stimuli or because some responded more to the object while others responded more to the panorama. To distinguish between the two possibilities, we analyzed the individual responses using the OPI (see Materials and Methods). Figure $2 D$ shows the distribution of the OPI values of 25 cells. If two groups of neurons were clearly defined, one with preference for objects and the other for optic flow fields, a bimodal distribution would be expected. The graph shows no indication of bimodal 

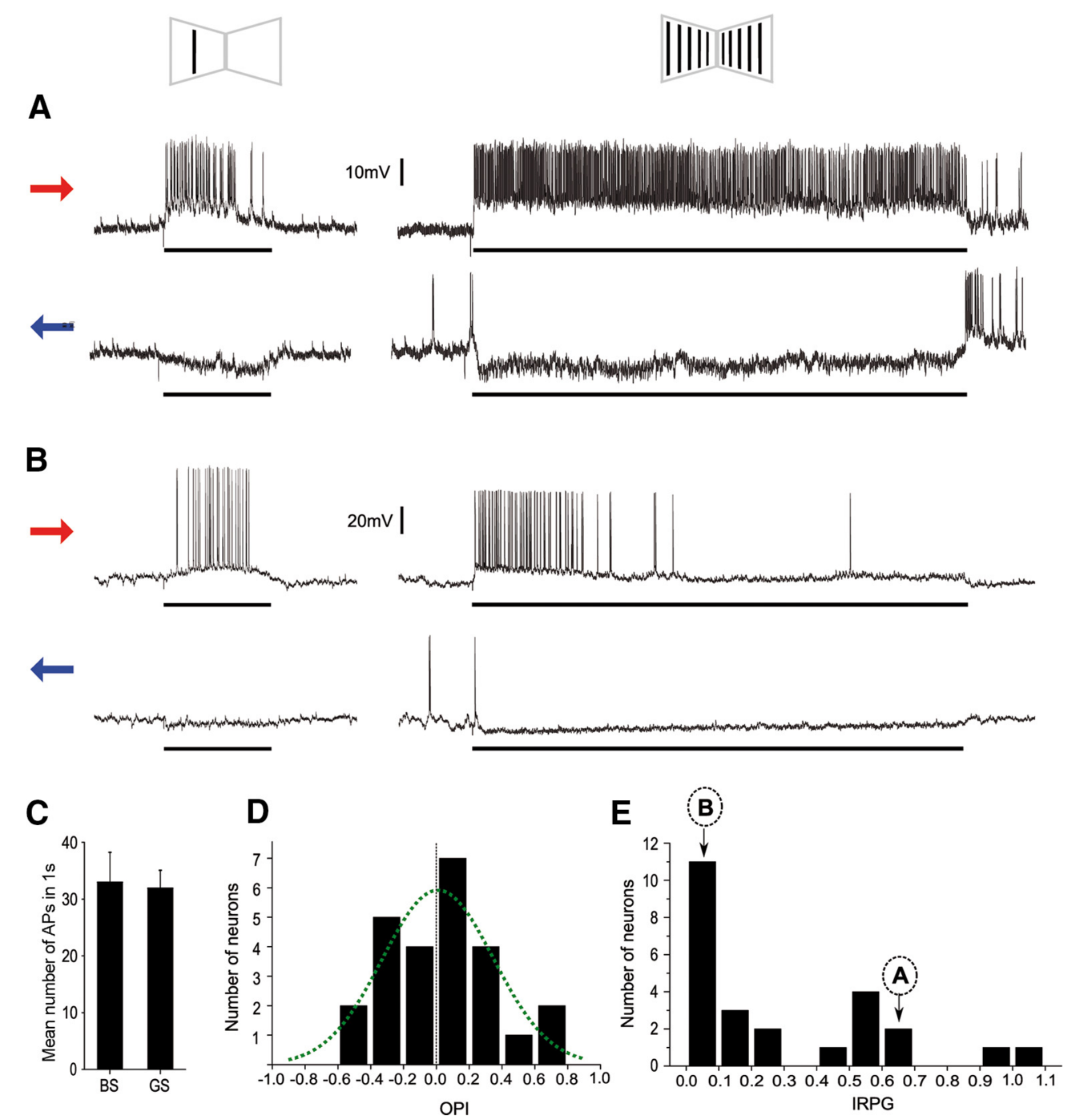

Figure 2. Response preference of $L C D C S$. $A, B$, Two examples of the types of responses found in the $L C D C S$. The left column shows responses to a bar (BS; see scheme above) moving from front to back (red arrow) or from back to front (blue arrow). The right column shows responses of the cells to a grating stimulus (GS) moving coherently on the two monitors. Both cells ( $A$ and $\boldsymbol{B}$ ) have the same direction preference, but while $\boldsymbol{A}$ shows a sustained response to the grating, $\boldsymbol{B}$ rapidly stops responding. Black rectangles beneath the recordings indicate the period of motion of the bar ( $3.36 \mathrm{~s})$ or the grating (15 s). C, The mean response triggered by the BS was similar to the one evoked by the GS; two-tailed paired $t$ test: $t_{(24)}=0.2617 ; p=0.7958$. Error bars indicate SEM. D, Distribution of LCDCS OPIs summarizing their preference to a BS compared with a GS. The index varies from 1 to -1 , corresponding to exclusive bar response or exclusive grating response, respectively. Values around 0 represent no preference. $E$, Distribution of LCDCS IRPGs summarizing the response persistence to the grating when the stimulus moved in the preferred direction. The distribution of data suggests that at least two distinct populations of neurons are contained in this sample. The majority shows IRPG close to 0 , indicating deep levels of adaptation; $n=25$. The localization of the neurons exemplified in $A, B$ is indicated by arrows.

distribution. In fact, data fitted to a normal distribution (Gaussian nonlinear regression, least fit squares, $F=18.27789$, Prob $>F=0.00202$; best-fit values: amplitude: 5.958; mean: 0.009112; SD: 0.3378; Fig. 2D). The majority of the cells, about half of our population sample, showed no preference between the two stimuli (OPI between 0.2 and -0.2). About a quarter of the cells responded more to the bar (OPI $>0.2)$, and the other quarter preferred the grating (OPI $<-0.2$ ). On the other hand, some of the cells showed a steady state response during the $15 \mathrm{~s}$ of grating stimulation (Fig. $2 A$ ), while others showed a quick adaptation (Fig. 2B). This suggests the existence of cells dedicated to serve different functions. We analyzed the adaptation phenomenon in 25 cells by using the IRPG (see Materials and Methods). Figure $2 E$ appears to separate at least two populations of neurons. On one side, 16 neurons that showed a deep level of adaptation (IRPG $<0.3$ ), with many that dropped to zero response at the end of the trial. On the other side, nine neurons that showed fairly sustained responses (IRPG $>0.4$ ), with two that showed absolutely no decay. For each neuron, a similar response dynamic (either sustained or adapted) was seen during the $15 \mathrm{~s}$ of stimulation in the preferred and in the null directions.

In the literature, DS neurons that respond to optic flow field rather than to object motion typically show a relatively sustained response to continuous stimulation (Rind, 1983, 1990; Maddess and Laughlin, 1985). Conversely, DS elements more reactive to target motion than to optic flow rapidly adapt with repeated stimulation (Medan et al., 2007; Glantz, 2008). Therefore, we expected that those cells showing a preference for the bar over the grating would be the ones adapting more rapidly to the grating. To test this, we evaluated the correspondence between OPI and the IRPG values of the neurons. Contrary to our expectations, there was no correlation $\left(R^{2}=-0.04195, p=0.85609\right)$.

\section{Location and morphology of LCDCs}

We stained 15 LCDCs, all of them interneurons of the optic lobe. Contrasting with all lobula tangential neurons so far identified in 

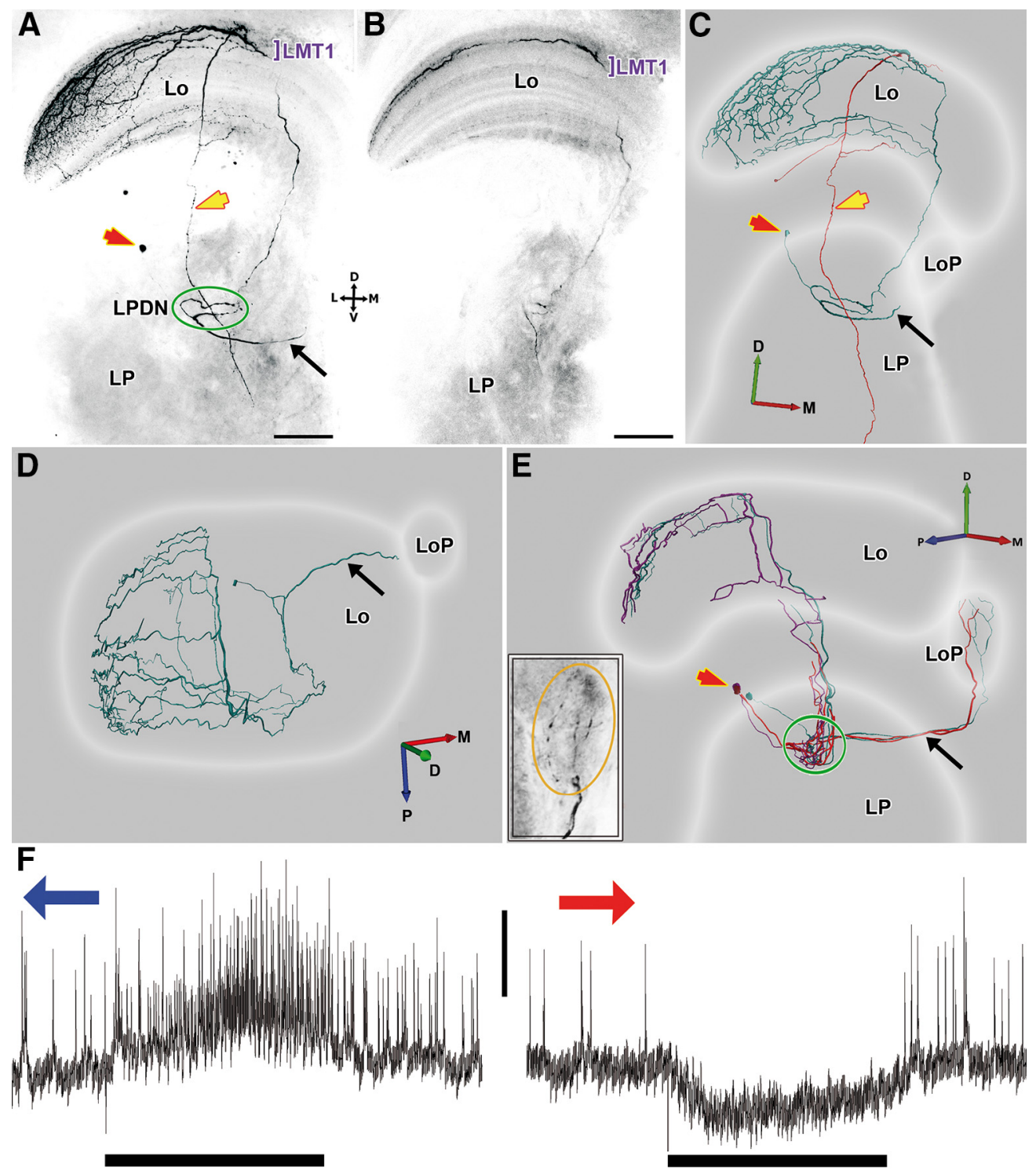

Figure 3. Two representative examples of $L C D C 1$ morphologic reconstructions. A, Z projection of a confocal stack of a whole mount preparation of the optic lobe showing an intracellularlystained neuron of the type LCDC1 arborizing in lobula (Lo) and lateral protocerebrum (LP). Given that the lobula has a curved structure in both anteroposterior and lateromedial axes, the branches seem to project to different layers of the lobula. However, this is an effect of collapsing the whole series of confocal images into a single picture. $\boldsymbol{B}, \mathbf{Z}$ projection of a substack of the confocal images from $\boldsymbol{A}$ showing that the tangential arborizations are present mainly in the first lateromedial tangential layer (LMT1). C, Anterior view of a three-dimensional reconstruction of the neuron showed in $\boldsymbol{A}$. The soma is located distal to the LP (red arrow), the neuron sends a branch toward the lobula plate (LoP; black arrow) and projects to a discrete region in the LP (green oval); the lateral protocerebrum directional neuropil, LPDN. Because of the presence of two cell bodies in several preparations, it could be determined that two neurons were co-stained: an LCDC1 (cyan) and a columnar neuron (red), whose axon descends through the lobula and the lateral protocerebrum to exit the optic lobe (yellow arrow). D, Same reconstruction, dorsal view. The arborization pattern in the lobula can be observed: a main neurite covers the lobula anteroposteriorly giving rise to several parallel secondary branches on the lateromedial axis, thinning toward the lateral side. $E$, Three-dimensional reconstruction of another preparation where three LCDCS1 were co-stained. Lateral view. LCDCs1 projections into the LoP are seen. The inset shows a z projection of a confocal substack of a whole mount preparation zooming in the branches in the LoP (orange oval). $\boldsymbol{F}$, Electrophysiological responses recorded from the neurons showed in $\boldsymbol{E}$ to a bar moving in its preferred and null direction, respectively. Blue arrow: back to front motion; red arrow: front to back motion. Bar beneath recording indicates the time frame when stimulus moves $(3.36 \mathrm{~s})$. Different sizes of APs can be observed, likely corresponding to electrical coupling among the three reconstructed neurons. Vertical scale bar: $10 \mathrm{mV}$. D: dorsal, V: ventral, L: lateral, M: medial, A: anterior, P: posterior. Scale bar: $100 \mu \mathrm{m}(\boldsymbol{A}, \boldsymbol{B})$.

the crab Neohelice, LCDCs do not project their axons along the protocerebral tract. All LCDCs presented profuse arborizations in the first lateromedial tangential layer (LMT1; Sztarker et al., 2005) of the lobula, sent a projection toward the lobula plate, and arborized in a previously undescribed region of the lateral protocerebrum that we termed here the lateral protocerebrum directional neuropil (see LPDN section below). Arborizations in the lobula and lobula plate probably constitute the input regions of these neurons while the LPDN would be the output region (the reasoning for this is presented in Discussion). Processes of
LCDCs within the lobula plate were observed in some but not all the preparations, likely because of a partial staining (Figs. $3 E$, $4 B$ ). However, all stained neurons presented a fiber projecting to the ventral surface of the lobula plate which followed a very direct trajectory toward this neuropil (Figs. $3 A, C-E, 4 B$, black arrows). Because no other defined structures are present in that area, we are confident that all LCDCs branch in the lobula plate. In the lobula, the main neurite is oriented in a postero-anterior axis, from which originate several secondary branches that run perpendicular to the main neurite, getting thinner as they 


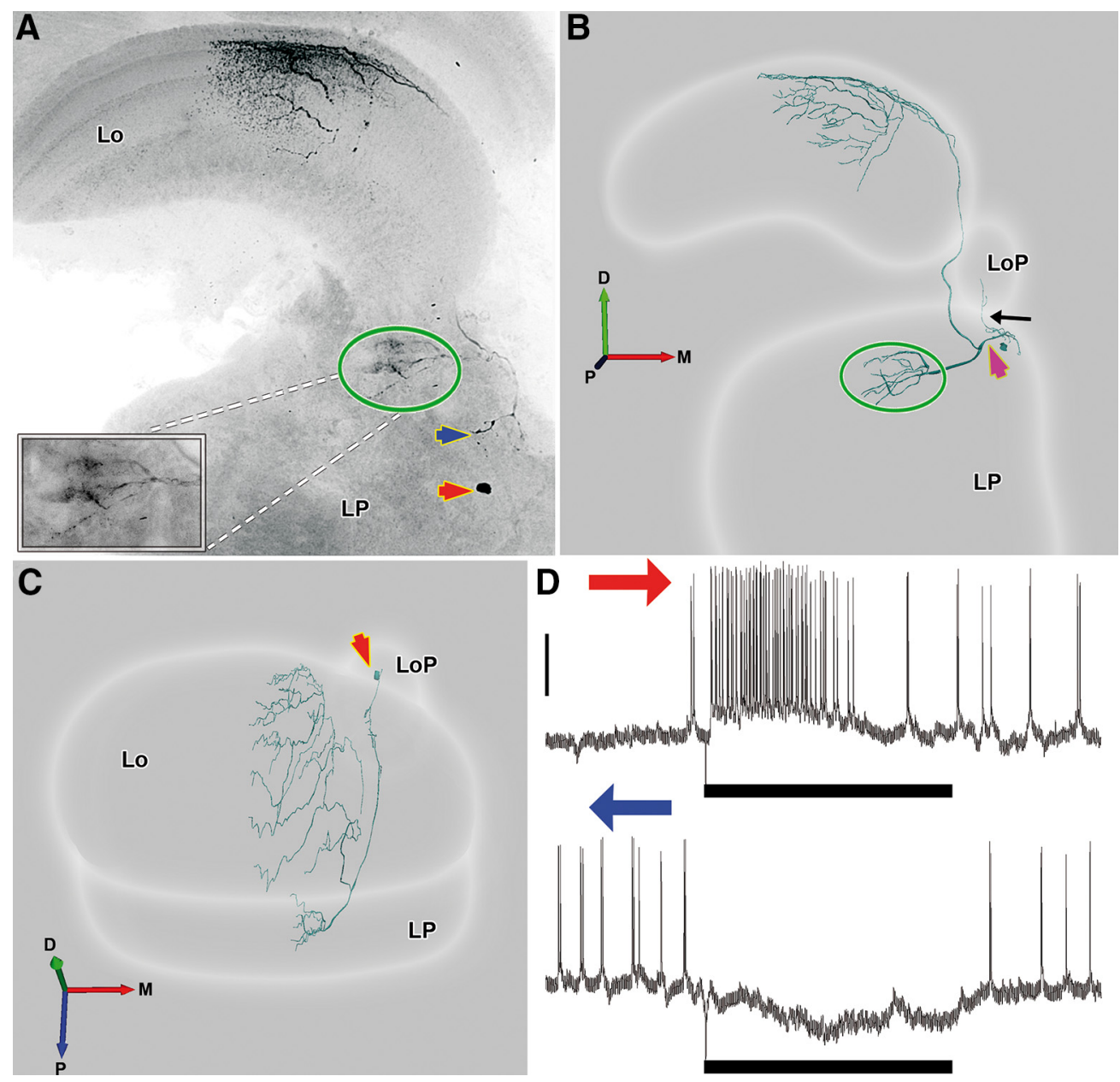

Figure 4. A representative example of $L C D C 2$ morphologic reconstruction. $A, Z$ projection of a confocal stack showing an intracellularly-stained neuron of the type $L C D C 2$. The soma is on a medial position (red arrows in $A-C$ and below the lobula plate (LoP). The neuron arborizes profusely in the first lateromedial tangential layer (LMT1), sends projections into the LoP and to a discrete region in the lateral protocerebrum (LP; green oval). The inset shows a detail of this area. $L C D C S 2$ also show a small arborization near the soma (blue arrow). $\boldsymbol{B}, \boldsymbol{C}$, Anterior and dorsal view, respectively, of a three-dimensional reconstruction of the neuron showed in $\boldsymbol{A}$. In the anterior view $(\boldsymbol{B})$, the fiber leading to the LoP (black arrow) and the fiber (magenta arrow) projecting to the lobula and to the protocerebral region surrounded by the green oval can be seen. $\boldsymbol{D}$, Electrophysiological responses recorded from the same neuron to a bar moving in its preferred (above) and null direction (below). Vertical bar: $20 \mathrm{mV}$. Blue arrow: back to front motion; red arrow: front to back motion. Bar beneath recording indicates the time frame when stimulus moves (3.36 s). D: dorsal, V: ventral, L: lateral, M: medial, A: anterior, P: posterior.

progress toward the lateral side. Although all cells shared these characteristics, we found consistent morphologic differences between reconstructions that led us to subdivide this group into two subcategories, the LCDCs of type 1 (Fig. 3) and the LCDCs of type 2 (Fig. 4).

LCDCs1 have their somata located near the dorsal surface of the lateral protocerebrum (Fig. $3 A, C, E$, red arrows). From the soma, the neurite enters the lateral protocerebrum and then bifurcates. One of the fibers sharply turns toward the lobula plate to arborize there (Fig. 3A,C-E, black arrow). The lobula plate is located anteriorly and medial to the lobula while the bifurcation of the neurite is in the posterior part of the lateral protocerebrum (Fig. 3D). This means that the fiber traverse almost the whole lateral protocerebrum to reach the base of the lobula plate (Fig. $3 E$ ). The other fiber goes into the LPDN through its lateral side and gives rise to several processes. At the other side of the LPDN, a single fiber leaves ascending toward the lobula where it branches profusely into the LMT1 (Fig. $3 A, B$ ). In this morphologic subtype co-staining of several units of the same type was seen in half of the preparations (Fig. 3E). A columnar neuron (Fig. 3C, red tracing) was also usually co-stained, apparently synapsing with the LCDC1 at the level of LMT1 (present in three out of four preparations). Its soma was near the lobula proximal surface, and its axon traversed the LPDN in its transit toward the protocerebral tract (Fig. 3A,C, yellow arrows).

LCDCs2 have their somata located toward the medial side of the lateral protocerebrum, approximately below the lobula plate (Fig. $4 A, C$, red arrows). From the soma, one fiber projects to the lobula plate (Fig. $4 B$, black arrow) and ramifies in thin secondary processes inside the lobula plate. Another fiber (Fig. 4B, magenta arrow) derived from the soma, which has higher caliper, arborizes in a small area in the lateral protocerebrum (Fig. $4 A$, blue arrow). Then, the fiber continues its trajectory toward the posterior part of the lateral protocerebrum (Fig. 4C) until it bifurcates. One fiber enters medially into the LPDN, where it arborizes profusely (Fig. $4 A, B$, green oval). The other fiber projects distally toward the lobula, giving rise to an extended dendritic field in LMT1 (Fig. $4 A, B$ ) that cover all the anteroposterior axis of the neuropil (Fig. 4C). At variance with the LCDC1 type, in the LCDC2 morphologic subtype, we never observed co-stained elements.

We looked hard to find a relationship between the two morphologic subtypes and physiological characteristics of the neurons, such as their level of spontaneous activity, size of spikes, maximum firing rate, or with characteristics of their responses 


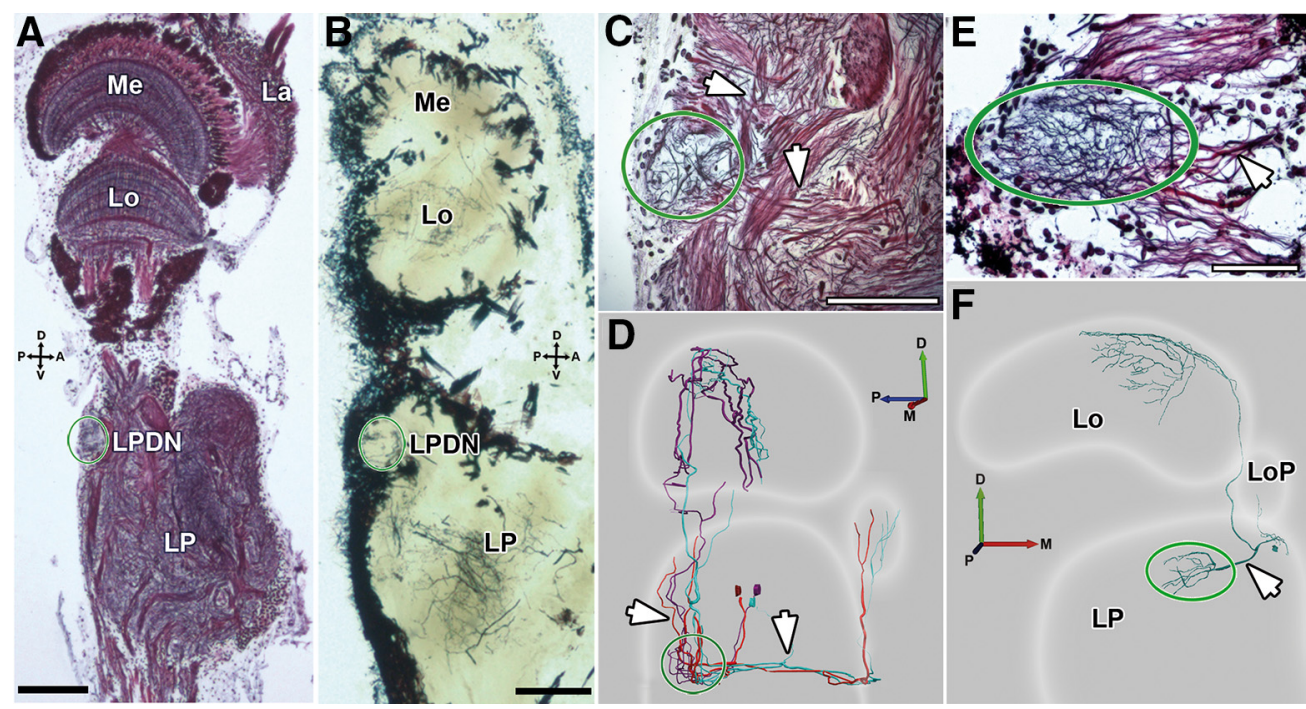

Figure 5. Lateral protocerebrum directional neuropil, LPDN. A, B, Longitudinal sections of the optic lobe stained with Bodian (thickness: $12 \mu \mathrm{m}$ ) or Golgi technique (thickness: $40 \mu \mathrm{m}$ ), respectively. The LPDN is situated on the dorso-posterior side of the lateral protocerebrum (green oval). C, Higher magnification of image $\boldsymbol{A}$ centered in the LPDN (green oval). In this transversal view, the LPDN is spherical and is connected to other regions (leading to the lobula and the lobula plate) by a dorsal and an anterior track (white arrows). $\boldsymbol{D}$, Three-dimensional reconstruction of LCDCS1 showing their projections to the LPDN (white arrows). These are probably the same projections showed in C. $\boldsymbol{E}$, Transversal view of a Bodian-stained section centered in the LPDN (green oval). In this orientation, the LPDN is elongated in the lateromedial axis. It receives neuronal projections from the medial side (white arrow). $\boldsymbol{F}$, Three-dimensional reconstruction of an LCDC2 where the connections with the LPDN can be seen (white arrow). This is probably the same bundle showed in E. D: dorsal, V: ventral, L: lateral, M: medial, A: anterior, P: posterior. Scale bar: $100 \mu \mathrm{m}(\boldsymbol{A}, \boldsymbol{B})$ and $50 \mu \mathrm{m}(\boldsymbol{C}, \boldsymbol{E})$.

such as their OPI and IRPG index values. However, we have not yet found any response parameter that allows us to reliably distinguish between the two morphologic types based on physiological differences.

\section{Lateral protocerebrum directional neuropil (LPDN)}

Because the two morphologic classes of LCDCs described here did arborize in a particularly well-defined structure of the lateral protocerebrum, we termed this structure the lateral protocerebrum directional neuropil, LPDN. The LPDN has a small volume $(50 \times 50 \times 100 \mu \mathrm{m}$ in the dorso-ventral, antero-posterior, and latero-medial axes, respectively), and it is located superficially in the dorso-posterior region of the lateral protocerebrum (Fig. $5 \mathrm{~A}$, $B$, green oval). Given its dimensions, the LPDN looks rather circular in longitudinal sections (Fig. $5 C$ ) and elongated in transversal views (Fig. 5E). In Bodian-stained longitudinal sections, the LPDN appears connected to other regions (leading to the lobula and the lobula plate) by two bundles (Fig. $5 C$, white arrows) that probably carry the processes of LCDCs1 (Fig. $5 D$ ). When seen in the transversal view, it looks connected to more medial regions of the lateral protocerebrum by only one bundle (Fig. $5 E$, white arrow), which is probably composed by LCDCs2 processes (Fig. $5 F)$.

\section{Discussion}

\section{Physiologic features of LCDCs}

The LCDCs described here for the first time present a remarkable directional selectivity for image motion exclusively in the horizontal plane, with a pronounced inhibitory response in the null direction. Most of these cells show characteristics compatible with a role in object motion detection: they present a small $\left(\sim 50^{\circ}\right)$ ipsilateral receptive field, strongly respond to a moving object, and rapidly adapt to a wide-field moving grating. Cells of similar characteristics have been recorded in a few insects (Collett, 1971, 1972; Olberg, 1981; Egelhaaf, 1985). Their function has been usually related with figure-ground discrimination or tracking behavior.

Some LCDCs, in contrast, exhibited intense and sustained responses to the grating (Fig. $2 A, E$ ), indicating their potential role in optic flow field processing. Interestingly, the two neurons that showed the most sustained responses to the grating (IRPG close to 1; Fig. $2 E$ ) had a front to back preferred direction. This is consistent with a recent study on the optomotor responses of this crab, which showed a robust front to back directional preference for optic flow when this stimulus is perceived monocularly (Barnatan et al., 2019). Worthy of note, front to back is also the preferred direction of neurons in the horizontal system (HS) involved in optomotor responses and course control in Diptera (HS tangential cells; Hausen, 1982a,b).

\section{Morphologic features of LCDCs}

Concerning LCDCs morphology, three things are important to highlight. First, unlike all other motion-sensitive LG cells previously characterized in crabs, whose axons project along the protocerebral tract toward the central brain (Berón de Astrada and Tomsic, 2002; Medan et al., 2007), LCDCs are large neurons internal to the optic lobe. Second, all stained units projected to the lobula plate, which is thought to be homologous to the dipteran lobula plate (Strausfeld, 2005, 2009; Bengochea et al., 2018). Third, all LCDCs arborize in an undescribed small neuropil of the lateral protocerebrum, here called LPDN. This neuropil resembles in shape the proximal promontory of the diamedullary neuropil described by Blaustein et al. (1988). Unfortunately, the structure has been poorly described in that account, and therefore, establishing a relation with the LPDN is premature. The LPDN may also bring to mind the protocerebral structures known as optic glomeruli, which have been defined as sites where an assembly of lobula complex neurons that are uniquely tuned to a set of visual parameters terminate (Strausfeld and Okamura, 2007). All the LCDCs were found to arborize in the 


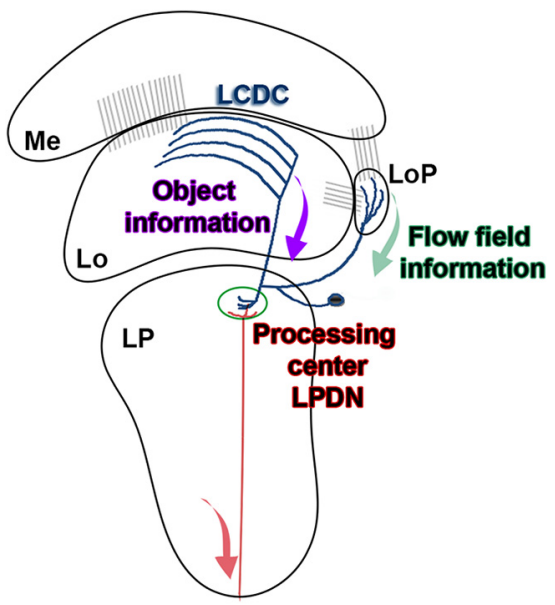

Figure 6. Working model of the information flow in the LCDCs system/LPDN. Schematic picture displaying the general structure of an LCDC with the proposed information flow. The lobula receives retinotopic information from the medulla and the lobula plate receives retinotopic information from both the medulla and the lobula (gray connections). Information about objects would be extracted from the lobula (purple arrow), while information about the movement of the panorama would be obtained from the lobula plate (green arrow). This information would converge in the LPDN to improve object discrimination or to command appropriate directional motor actions (pursuit or avoidance) triggered by visual stimuli. Me: medulla; Lo: lobula; LoP: lobula plate; LP: lateral protocerebrum.

LPDN; however, we do not know whether this is the output site of these neurons (but see below in this section). In addition, the neurons ending in the optic glomeruli are columnar neurons, while LCDCs are wide-field tangential neurons. These two reasons also prevent us from using the term optic focus for the structure described in this article (Strausfeld and Nassel, 1981). Instead, we opted to refer to this structure as the LPDN.

The two morphologic types of LCDCs described here differ in the position of the soma, the orientation in which they enter the LPDN, and the probability to be co-stained with other neurons. However, there is a high consistency between the sites of synaptic contact of the two types of LCDCs, with both types presenting conspicuous arborizations in layer 1 of the lobula, in the lobula plate, and in the LPDN. The, fact that the two types share the sites where they collect and convey information suggests they might be involved in equivalent computations. Consistent with this, we found no obvious differences between the physiological properties of the two morphologic types. Even within the subgroup of nine units that showed a maintained response to the grating (Fig. 2E), of which we stained three units, two of them showed a LCDC1 and one showed a LCDC2 morphology. It is possible that the stimulus preferences that distinguish the two morphologic types are not the ones that we have used in the present study. Further studies including additional features of image motion, such as contrast, size, velocity, or target to background relation may help to tell apart the two groups.

At present, we cannot be certain about the direction in the flow of information within LCDCs. The scheme in Figure 6 represents our heuristic hypothesis based on the information we currently have. Because both the lobula and the lobula plate receive retinotopic information (Berón de Astrada et al., 2013; Bengochea and Berón de Astrada, 2014; Bengochea et al., 2018; Fig. 6, gray connections), it is reasonable to assume that the arborizations in these areas are input regions while the branches within the LPDN, where retinotopy is not observed, would be the output region. Compatible with this hypothesis, preliminary results indicate that similar to MLG1 neurons (Medan et al., 2015), there is a correspondence between the position of the arborization of individual LCDCs along the lateromedial axis of the lobula (the axis that maps azimuthal positions) and the azimuthal location of their receptive fields.

What kind of information could be integrated in the LPDN? It is appealing to think that the LPDN is a convergence center where information about the direction of moving objects is integrated with information about optic flow field motion (Fig. 6) to help object discrimination or to command appropriate directional motor actions (pursuit or avoidance) triggered by visual stimuli.

\section{Why LCDCs are only sensitive to horizontal motion?}

LCDCs have a preference for horizontally moving objects (Fig. 1). In fact, we could not find any DS neuron with a stark vertical preference. Previous work on Neohelice shows that the lobula contains different classes of LG neurons that collect information from the retinotopic columnar mosaic and project their axons to the midbrain (Berón de Astrada and Tomsic, 2002; Medan et al., 2007, 2015). The LG neurons respond more to the motion of a bar than to a GS and show a rapid reduction in the number of stimulus-elicited spikes on repeated stimulation, which temporal course and persistence parallel the changes occurring in the escape behavior of the crab (Tomsic et al., 2003; Medan et al., 2007). LG neurons have no direction or orientation preference, except for MLG1 that show a slight preference for horizontal motion and respond more in one direction (Medan et al., 2007). Nonetheless, the directional preference is exhibited by a moderate difference in the intensity of response but not by responses of opposite sign as it is evident in the LCDCs (Fig. $1 A-C$ ). The same holds true for horizontal versus vertical motion preference. Thus, although less noticeable than in the LCDCs described here, other neurons from the lobula of the crab exhibit preference for horizontal motion.

The lobula plate tangential cells (LPTCs) of Diptera are organized in two systems, distinguished by their preferred motion axis, a HS and a vertical system (VS). In fast flying insects, the two systems are critical for detecting course deviations in the yaw, pitch, and roll axes, which allows to rapidly implement the maneuvers that lead to course corrections (e.g., in Diptera: Krapp and Hengstenberg, 1996; Borst and Haag, 2002). For slow flying insects like butterflies or for walking animals like crabs, a VS may not be as necessary. In fact, in such animals, recordings of DS neurons with vertical preference is much less common (e.g. Heliconius erato: Swihart, 1968; Podophthalmus vigil crab: Waterman et al., 1964; Carcinus maenas crab: Sandeman et al., 1975; present results in Neohelice). Semiterrestrial crabs, like Neohelice, inhabit mudflat areas where they continually interact with other crabs that move along the horizontal plane. The most frequent interactions include chasing after smaller crabs, escaping from or fighting other crabs, and courtship, all of these visually-guided activities occurring at the level of the horizon. Therefore, from the perspective of vision, Neohelice lives in a vertically-compressed world, where most object motion corresponds to the movements of neighboring crabs along the horizontal plane (Tomsic et al., 2017). Besides, the fact that crabs locomote exclusively in two dimensions (except when they get into the burrow where vision becomes irrelevant) means that they only experience self-generated 
optic flow in the horizontal plane. Recent morphologic data from the lobula plate, the candidate region thought to analyze flow field motion in crabs, provide support for this notion. According to the number of subtypes and the morphology of medullary columnar cells reaching the lobula plate of Neohelice, it was proposed that this neuropil contains two instead of four functional directional layers as its dipteran counterpart. These two functional layers are thought to process opposite directions in the horizontal plane (Bengochea et al., 2018).

We have previously found optical and neuronal features that represent adaptations to the flat environment inhabited by this crab: they present a rim of maximum acuity around the equator of the eye (Astrada et al., 2012) and a vertical constriction of the receptive fields of the MLG1 system. This system is formed by an ensemble of 16 units that, together, cover the entire $360^{\circ}$ of the azimuthal plane, but in the vertical plane, all units have their receptive field centered over the eye equator (Medan et al., 2015). The results presented here showing that LCDCs are exclusively responsive to horizontal motion is another, perhaps the clearest, example of a neural adaptation of living in a flat environment.

\section{References}

Astrada MB, Bengochea M, Medan V, Tomsic D (2012) Regionalization in the eye of the grapsid crab Neohelice granulata (=Chasmagnathus granulatus): variation of resolution and facet diameters. J Comp Physiol A Neuroethol Sens Neural Behav Physiol 198:173-180.

Barnatan Y, Tomsic D, Sztarker J (2019) Unidirectional optomotor responses and eye dominance in two species of crabs. Front Physiol 10:586.

Barnett PD, Nordström K, O'Carroll DC (2007) Retinotopic organization of small-field-target-detecting neurons in the insect visual system. Curr Biol 17:569-578.

Bengochea M, Berón de Astrada M (2014) Organization of columnar inputs in the third optic ganglion of a highly visual crab. J Physiol Paris 108:6170.

Bengochea M, Berón de Astrada M, Tomsic D, Sztarker J (2018) A crustacean lobula plate: morphology, connections, and retinotopic organization. J Comp Neurol 526:109-119.

Berón de Astrada M, Sztarker J, Tomsic D (2001) Visual interneurons of the crab Chasmagnathus studied by intracellular recordings in vivo. J Comp Physiol A Neuroethol Sens Neural Behav Physiol 187:37-44.

Berón de Astrada M, Tomsic D (2002) Physiology and morphology of visual movement detector neurons in a crab (Decapoda: brachyura). J Comp Physiol A Neuroethol Sens Neural Behav Physiol 188:539-551.

Berón de Astrada M, Tuthill JC, Tomsic D (2009) Physiology and morphology of sustaining and dimming neurons of the crab Chasmagnathus granulatus (Brachyura: grapsidae). J Comp Physiol A Neuroethol Sens Neural Behav Physiol 195:791-798.

Berón de Astrada M, Bengochea M, Sztarker J, Delorenzi A, Tomsic D (2013) Behaviorally related neural plasticity in the arthropod optic lobes. Curr Biol 23:1389-1398.

Blaustein DN, Derby CD, Simmons RB, Beall AC (1988) Structure of the brain and medulla terminalis of the spiny lobster Panulirus argus and the crayfish Procambarus clarkii, with an emphasis on olfactory centers. J Crustac Biol 8:493-519.

Borst A, Haag J (2002) Neural networks in the cockpit of the fly. J Comp Physiol A Neuroethol Sens Neural Behav Physiol 188:419-437.

Collett T (1971) Visual neurones for tracking moving targets. Nature 232:127-130.

Collett T (1972) Visual neurones in the anterior optic tract of the privet hawk moth. J Comp Physiol 78:396-433.

DeVoe RD, Kaiser W, Ohm J, Stone LS (1982) Horizontal movement detectors of honeybees: directionally-selective visual neurons in the lobula and brain. J Comp Physiol A Neuroethol Sens Neural Behav Physiol 147:155170.

Egelhaaf M (1985) On the neuronal basis of figure-ground discrimination by relative motion in the visual system of the fly. Biol Cybern 52:195-209.
Gilbert C, Strausfeld NJ (1991) The functional organization of male-specific visual neurons in flies. J Comp Physiol A Neuroethol Sens Neural Behav Physiol 169:395-411.

Glantz RM (2008) Polarization vision in crayfish motion detectors. J Comp Physiol A Neuroethol Sens Neural Behav Physiol 194:565-575.

Hausen K (1982a) Motion sensitive interneurons in the optomotor system of the fly. Biol Cybern 46:67-79.

Hausen K (1982b) Motion sensitive interneurons in the optomotor system of the fly. Biol Cybern 45:143-156.

Hausen K, Egelhaaf M (1989) Neural mechanisms of visual course control in insects. In: Facets of vision (Stavenga DG, ed), pp 391-424. Berlin: Springer.

Ibbotson MR (1991) Wide-field motion-sensitive neurons tuned to horizontal movement in the honeybee, Apis mellifera. J Comp Physiol A Neuroethol Sens Neural Behav Physiol 168:91-102.

Krapp H, Hengstenberg R (1996) Estimation of self-motion by optic flow processing in single visual interneurons. Nature 384:463-466.

Maddess T, Laughlin SB (1985) Adaptation of the motion-sensitive neuron $\mathrm{H} 1$ is generated locally and governed by contrast frequency. Proc R Soc Lond B Biol Sci 225:251-275.

Medan V, Oliva D, Tomsic D (2007) Characterization of lobula giant neurons responsive to visual stimuli that elicit escape behaviors in the crab Chasmagnathus. J Neurophysiol 98:2414-2428.

Medan V, Berón De Astrada M, Scarano F, Tomsic D (2015) A network of visual motion-sensitive neurons for computing object position in an arthropod. J Neurosci 35:6654-6666.

Nordström K, Barnett PD, O'Carroll DC (2006) Insect detection of small targets moving in visual clutter. PLoS Biol 4:e54.

O'Carroll D (1993) Feature-detecting neurons in dragonflies. Nature 362:541-543.

Olberg RM (1981) Object- and self-movement detectors in the ventral nerve cord of the dragonfly. J Comp Physiol 141:327-334.

Rind FC (1983) A directionally sensitive motion detecting neurone in the brain of a moth. J Exp Biol 102:253-271.

Rind FC (1990) Identification of directionally selective motion-detecting neurones in the locust lobula and their synaptic connections with an identified descending neurone. J Exp Biol 149:21-43.

Romano A, Locatelli F, Freudenthal R, Merlo E, Feld M, Ariel P, Lemos D, Federman N, Fustiñana MS (2006) Lessons from a crab: molecular mechanisms in different memory phases of Chasmagnathus. Biol Bull 210:280-288.

Sandeman DC, Kien J, Erber J (1975) Optokinetic eye movements in the crab, Carcinus maenas. J Comp Physiol 101:259-274.

Scarano F, Tomsic D (2014) Escape response of the crab Neohelice to computer generated looming and translational visual danger stimuli. J Physiol Paris 108:141-147.

Strausfeld NJ (2005) The evolution of crustacean and insect optic lobes and the origins of chiasmata. Arthropod Struct Dev 34:235-256.

Strausfeld NJ (2009) Brain organization and the origin of insects: an assessment. Proc Biol Sci 276:1929-1937.

Strausfeld NJ, Nassel DR (1981) Neuroarchitecture of brain regions that subserve the compound eyes of crustacea and insect. In: Handbook of sensory physiology (Autrum H, ed), pp 1-132. Berlin: Springer.

Strausfeld NJ, Okamura JY (2007) Visual system of calliphorid flies: organization of optic glomeruli and their lobula complex efferents. J Comp Neurol 500:166-188.

Swihart SL (1968) Single unit activity in the visual pathway of the butterfly Heliconius erato. J Insect Physiol 14:1589-1601.

Sztarker J, Tomsic D (2008) Neuronal correlates of the visually elicited escape response of the crab Chasmagnathus upon seasonal variations, stimuli changes and perceptual alterations. J Comp Physiol A Neuroethol Sens Neural Behav Physiol 194:587-596.

Sztarker J, Tomsic D (2011) Brain modularity in arthropods: individual neurons that support "what" but not "where" memories. J Neurosci 31:81758180.

Sztarker J, Strausfeld NJ, Tomsic D (2005) Organization of optic lobes that support motion detection in a semiterrestrial crab. J Comp Neurol 493:396-411.

Tomsic D, Sztarker J (2019) Crustacean visual circuits underlying behavior. In: Oxford Research Encyclopedia of Neuroscience. New York: Oxford University Press. 
Tomsic D, Berón de Astrada M, Sztarker J (2003) Identification of individual neurons reflecting short- and long-term visual memory in an arthropodo. J Neurosci 23:8539-8546.

Tomsic D, de Astrada MB, Sztarker J, Maldonado H (2009) Behavioral and neuronal attributes of short- and long-term habituation in the crab Chasmagnathus. Neurobiol Learn Mem 92:176-182.

Tomsic D, Sztarker J, Berón de Astrada M, Oliva D, Lanza E (2017) The predator and prey behaviors of crabs: from ecology to neural adaptations. J Exp Biol 220:2318-2327.

Trischler C, Boeddeker N, Egelhaaf M (2007) Characterisation of a blowfly male-specific neuron using behaviourally generated visual stimuli. J Comp Physiol A Neuroethol Sens Neural Behav Physiol 193:559-572.

Warzecha AK, Egelhaaf M, Borst A (1993) Neural circuit tuning fly visual interneurons to motion of small objects. I. Dissection of the circuit by pharmacological and photoinactivation techniques. J Neurophysiol 69:329-339.
Waterman TH, Wiersma CA, Bush BM (1964) Afferent visual responses in the optic nerve of the crab, Podophthalmus. J Cell Comp Physiol 63:135155 .

Wicklein M, Varjú D (1999) Visual system of the European hummingbird hawkmoth Macroglossum stellatarum (Sphingidae, Lepidoptera): motion-sensitive interneurons of the lobula plate. J Comp Neurol 408:272-282.

Wiersma CA, Yanagisawa K (1971) On types of interneurons responding to visual stimulation present in the optic nerve of the rock lobster, Panulirus interruptus. J Neurobiol 2:291-309.

Yamawaki Y (2019) Unraveling the functional organization of lobula complex in the mantis brain by identification of visual interneurons. J Comp Neurol 527:1161-1178

Zeil J, Nalbach G, Nalbach HO (1989) Spatial vision in a flat world: optical and neural adaptations in arthropods. In: Neurobiology of sensory systems, pp 123-137. New York: Plenum Press. 